\title{
Caracterización de la práctica de una profesora al implementar un diseño sobre la función exponencial que integra GeoGebra
}

\author{
Karen Gisel Campo-Meneses ${ }^{1}$ \\ karencampo@uagro.mx \\ https://orcid.org/0000-0001-7483-3134 \\ Gilbert Andrés Cruz Rojas ${ }^{2}$ \\ gilbert.a.cruz.r@ correounivalle.edu.co \\ https://orcid.org/0000-0001-7391-9462 \\ ${ }^{1}$ Universidad Autónoma de Guerrero (UAGro, México) \\ ${ }^{2}$ Universidad del Valle (Univalle, Colombia)
}

Recibido: 28/04/2020 Aceptado: 27/07/2020

\section{Resumen}

Este artículo reporta la caracterización de la práctica de enseñanza de una profesora cuando implementa un diseño de tareas sobre a la función exponencial que integra GeoGebra. Para la configuración del diseño de tareas y el análisis de la implementación, se tuvo en cuenta como aproximación teórica la Orquestación Instrumental, particularmente sus dimensiones, elementos y tipos. Para llevar a cabo la investigación se empleó el estudio de casos en el que participó una profesora de matemáticas. El análisis muestra que, en su mayoría, las decisiones tomadas por la profesora en la implementación del diseño contribuyeron, de alguna manera, en el desarrollo de las tareas por parte de los estudiantes y en la exposición y evaluación de las estrategias y razonamientos empleados por ellos. Así pues, aunque el diseño esté estructurado, este es susceptible a modificaciones por parte del profesor según el contexto de implementación y sus intenciones didácticas, siempre y cuando no se aleje de los objetivos propuestos en su configuración didáctica.

Palabras clave: Función exponencial; Orquestación instrumental; GeoGebra.

\section{Characterization of a teacher's practice when implementing a design on the exponential function that integrates GeoGebra}

\begin{abstract}
This article reports the characterization of the teaching practice of a teacher when she implements a task design on the exponential function that integrates GeoGebra. For the configuration of the task design and the analysis of the implementation, the Instrumental Orchestration was taken into account as a theoretical approach, particularly its dimensions, elements and types. To carry out the research, the case study was used, in which a mathematics teacher participated. The analysis shows that, for the most part, the decisions made by the teacher in the implementation of the design contributed, in some way, in the development of the tasks by the students and in the exposition and evaluation of the strategies and reasoning. employed by them. Thus, although the design is structured, it is susceptible to modifications by
\end{abstract}


the teacher according to the context of implementation and its didactic intentions, as long as it does not depart from the objectives proposed in its didactic configuration.

Keywords: Exponential function; Instrumental orchestration; GeoGebra.

\title{
Caracterização da prática do professor ao implementar um projeto na função exponencial que integra o GeoGebra
}

\begin{abstract}
Resumo
Este artigo relata a caracterização da prática pedagógica de uma professora ao implementar um desenho de tarefa na função exponencial que integra o GeoGebra. Para a configuração do desenho da tarefa e análise da implementação, a Orquestração Instrumental foi considerada como uma abordagem teórica, particularmente as suas dimensões, elementos e tipos. Para a realização da pesquisa, foi utilizado o estudo de caso do qual participou uma professora de matemática. A análise mostra que, na sua maioria, as decisões tomadas pela professora na implementação do desenho contribuíram, de alguma forma, no desenvolvimento das tarefas pelos alunos e na apresentação e avaliação das estratégias e raciocínios utilizados pelos eles. Assim, embora o projeto seja estruturado, é suscetível de modificações por parte do professor de acordo com o contexto de implementação e suas intenções didáticas, desde que não se desvie dos objetivos propostos em sua configuração didática.
\end{abstract}

Palavras-chave: Função exponencial; Orquestração instrumental; GeoGebra.

\section{Introducción}

La función exponencial ha sido objeto de estudio en diversas investigaciones, las cuales se han realizado desde diferentes enfoques: registros de representaciones semióticas (Castro et al., 2017; Sureda \& Otero, 2013); aplicaciones de las funciones exponenciales (Sastre et al., 2017); desarrollo del razonamiento covariacional (Ellis et al., 2016; Trejo \& Ferrari, 2018), y diseños de actividades con tecnología (Castillo et al., 2013), entre otros.

Respecto a las investigaciones que se han enfocado en los registros de representaciones semióticas, se encuentra la investigación realizada por Castro et al. (2017), quienes estudiaron los efectos cognitivos de los diversos cambios de registros de representación de la función exponencial en los estudiantes de Cálculo Diferencial. Sureda y Otero (2013), también se enfocan en los registros de representación, pero describiendo el proceso de conceptualización de la función exponencial e identificando cinco niveles relacionados con los registros de representación empleados por los estudiantes. Estos mismos autores mencionan que la conceptualización de la función exponencial es una tarea compleja que no se realiza en todos 
los sistemas de representación al mismo tiempo y, sugieren la existencia de una progresividad en la conceptualización, que va desde los esquemas lineales hasta los exponenciales vinculados con los sistemas de representación. La explicitación, discusión y formulación de los conceptos en cada sistema resulta de gran importancia en la transición de lo lineal a lo exponencial.

En las investigaciones que se centran es las aplicaciones de las funciones exponenciales, se cita la investigación de Sastre et al. (2017), quienes estudiaron las aplicaciones de estas funciones en diversos fenómenos de la vida real y de otras ciencias. En esta investigación muestran un diverso panorama en el que se pueden modelar fenómenos usando las funciones exponenciales. Entre las aplicaciones existen las siguientes: crecimiento poblacional, estudio del pH, el consumo de energía, el consumo de los recursos naturales, la desintegración radiactiva, el interés compuesto de un capital, entre otros.

Entre las investigaciones que se han enfocado en estudiar el desarrollo del razonamiento covariacional exponencial (tercer grupo) está la realizada por Ellis et al. (2016) quienes presentan una Trayectoria de Aprendizaje de Crecimiento Exponencial que identifica y caracteriza las actividades iniciales para desarrollar la comprensión del crecimiento exponencial como resultado de un énfasis de instrucción en la covariación a partir de dos experimentos de enseñanza. Como resultado de su investigación, obtuvieron que los estudiantes logran reconocer el crecimiento exponencial encontrando el factor de crecimiento y la relación funcional $y=b^{x}$. Sin embargo, mostraron dificultad para razonar de manera continua, para lo cual estos autores sugieren que a los estudiantes se les debe proporcionar oportunidades para razonar a través de tareas que les permitan reflexionar y solidificar su pensamiento, además de empezar a trabajar desde la escuela ideas de crecimiento exponencial y explorar cómo estas cantidades varían.

Otra investigación es la de Trejo y Ferrari (2018) quienes, desde este mismo enfoque covariacional, realizan un acercamiento al concepto de función exponencial, mediante una construcción de puntos en GeoGebra con estudiantes del bachillerato mexicanos y como resultado encontraron que los estudiantes lograron identificar dos variaciones distintas, una para los valores de $x$ y otra para los valores de $y$, sin embargo, se les dificultó percibir la coexistencia y la codependencia.

Finalmente, en las investigaciones direccionadas a realizar diseños de actividades con tecnología se encuentra la investigación de Castillo et al. (2013), quienes diseñan una secuencia 
de aprendizaje para caracterizar la familia de curvas correspondiente a la función $f(x)=e^{a x}$, en relación con los efectos geométricos de deformación y reflexión visualizados tras la variación del parámetro contenido en la expresión de la función, utilizando GeoGebra. En esta investigación se puntualiza que a través del uso adecuado de un deslizador se pueden establecer relaciones entre los valores del parámetro (en intervalos establecidos convenientemente) y las curvas representadas en la vista gráfica del programa, lo cual dota de sentido a las transformaciones analizadas en la secuencia. Además, el trabajo con GeoGebra permite establecer conexiones entre las principales representaciones de las funciones (expresiones algebraicas y gráficas) utilizadas en la secuencia, lo que conlleva a desarrollar la comprensión de los efectos analizados con mayor profundidad.

Como se muestra de manera general, en las investigaciones citadas, se reportan dificultades por parte de los estudiantes para comprender la función exponencial. Los diseños elaborados se han centrado en los estudiantes y muestran que aún quedan opciones por indagar sobre cómo contribuir para que los estudiantes logren comprender esta función. En este sentido, se considera importante realizar más diseños para el aula y que se centren tanto en la práctica del profesor como en los estudiantes, ya que es el profesor quien debe orquestar la clase a fin de que se cumplan los objetivos de aprendizaje, además de involucrar herramientas tecnológicas que permitan de alguna manera promover el desarrollo de diferentes habilidades en los estudiantes y les facilite el análisis de diferentes representaciones de la función y sus respectivos parámetros.

Además, no se encuentran artículos en la literatura enfocados en la práctica del profesor cuando integra GeoGebra en la enseñanza de las funciones exponencial y logarítmica, empleando la orquestación instrumental, lo cual sería el aporte de esta investigación. Así pues, este artículo tiene por objetivo caracterizar la práctica de una profesora cuando implementa un diseño de tareas sobre la función exponencial e integrando GeoGebra. Se eligió este software porque es considerado como una herramienta didáctica de fácil acceso (es un software libre) y manipulación. A través de su uso es posible dibujar y explorar gráficas de funciones vinculándolas con otras representaciones semióticas como tablas de valores y expresiones algebraicas (Castillo et al., 2013). 
Además, como lo señala Santos Trigo (2019), el uso de GeoGebra proporciona un conjunto de posibilidades, que el profesor puede usar, para que los estudiantes conceptualicen y representen objetos matemáticos y tareas dinámicamente. El análisis de los comportamientos de los parámetros u objetos dentro de la configuración podría llevar a los estudiantes a identificar propiedades para apoyar las relaciones matemáticas emergentes.

Desde la perspectiva teórica que se define en el presente estudio se reconoce la importancia de investigaciones en este marco para el estudio y entendimiento de la práctica de enseñanza en cierto modo, lo cual tiene en cuenta la forma en que el profesor integra un instrumento en su clase, permitiéndole a los estudiantes concebir las tareas matemáticas como una oportunidad para participar en actividades matemáticas que incluyan la construcción de modelos dinámicos, la formulación de conjeturas y busquen diferentes argumentos para apoyarlas.

\section{Referentes teóricos}

La integración de tecnología digital en las prácticas de enseñanza es un tema que ha generado un incremento en cantidad de investigaciones que se realizan en el campo de la Educación Matemática. Este incremento puede observarse, en las líneas de investigación de las memorias del Congress of the European Society for Research in Mathematics Education (CERME), Psychology Mathematics Education (PME) y North American Chapter of the International Group for the Psychology of Mathematics Education (PME-NA). Se considera que las tecnologías digitales permiten la exploración de otros campos que dan lugar a la visualización, el dinamismo y la integración de diferentes representaciones. Sin embargo, la integración de tecnologías digitales es un tema complejo que cuestiona las metas de la Educación Matemática y de las prácticas de enseñanza (Trouche \& Drijvers, 2014).

Tal complejidad ha llevado a indagar sobre cómo se ven modificadas las prácticas de enseñanza y sus objetivos cuando se integra tecnología en el aula. Así el enfoque instrumental es una de las teorías que permite dar cuenta de esta complejidad, como los estudios realizados por Pepin et al., (2017), Clivaz y Shuilleabhain (2017) y Artigue (2019). 


\section{Enfoque instrumental}

El enfoque instrumental tiene sus raíces en la ergonomía cognitiva planteada por Verillon y Rabardel en 1995 y la teoría antropológica de la didáctica de Chevallard en 1999 (Trouche \& Drijvers, 2014) y resalta tres aspectos: la relación significativa entre una herramienta y su facilidad para llevar a cabo una tarea específica; el papel del profesor, quien orquesta la clase y por tanto juega un papel fundamental, y la conceptualización, que es concebida como un proceso de orden mediante el cual se tiene en cuenta conscientemente toda la información disponible, tanto de algún artefacto tecnológico como de otras fuentes, buscando así la coherencia matemática entre ellas (Trouche \& Drijvers, 2014).

En este enfoque instrumental se considera que el uso de una herramienta tecnológica en el aula, implica un proceso de génesis instrumental (Drijvers et al., 2010). Esta enuncia el proceso mediante el cual un artefacto (entendido como un objeto material o simbólico) se convierte en instrumento (es una construcción del usuario que está compuesto por el artefacto y los esquemas puestos en juego cuando se resuelve alguna tarea). En este sentido Trouche (2002) plantea que la génesis instrumental es un proceso complejo que implica dos movimientos interrelacionados: la instrumentalización y la instrumentación. La primera referida al artefacto, a sus características, formas de uso, estructuras y funcionamiento. Y la segunda, referida al sujeto, a la forma en que usa el artefacto, los esquemas que emergen cuando se enfrenta a ciertas tareas, su evolución y la respectiva asimilación de nuevos artefactos y acomodación de los esquemas constituidos.

En este proceso el papel del profesor es fundamental, ya que no basta solo con involucrar los artefactos al proceso educativo, sino la forma en que como orquestador de la clase, debe permitir que los artefactos involucrados puedan llegar a ser instrumentos en la actividad matemática. Así, en este trabajo se emplea los elementos del enfoque instrumental que a continuación se abordan.

\section{Orquestación instrumental}

La orquestación instrumental es definida como la gestión didáctica del sistema de instrumentos a cargo del profesor. Esto implica que el profesor tenga en cuenta los artefactos 
(computadores, lápiz y papel, GeoGebra, calculadoras, entre otros) que se van a integrar en la clase y su respectiva intencionalidad didáctica a fin de que proporcione construcciones mentales en los estudiantes sobre los conceptos matemáticos abordados, además de tomar decisiones respecto al diseño de la clase y a los criterios que orientan el diseño de tareas (Trouche, 2002). En otras palabras, Trouche (2002) afirma que la noción de orquestación instrumental permite estudiar un proceso colectivo, en el que el profesor propone una organización particular de la clase que favorece la actividad matemática. De acuerdo con Drijvers et al. (2010), en la orquestación instrumental se reconocen elementos, dimensiones y tipos de orquestación instrumental como se muestra en la Figura 1.

Figura 1: Dimensiones, tipos y elementos de la orquestación instrumental

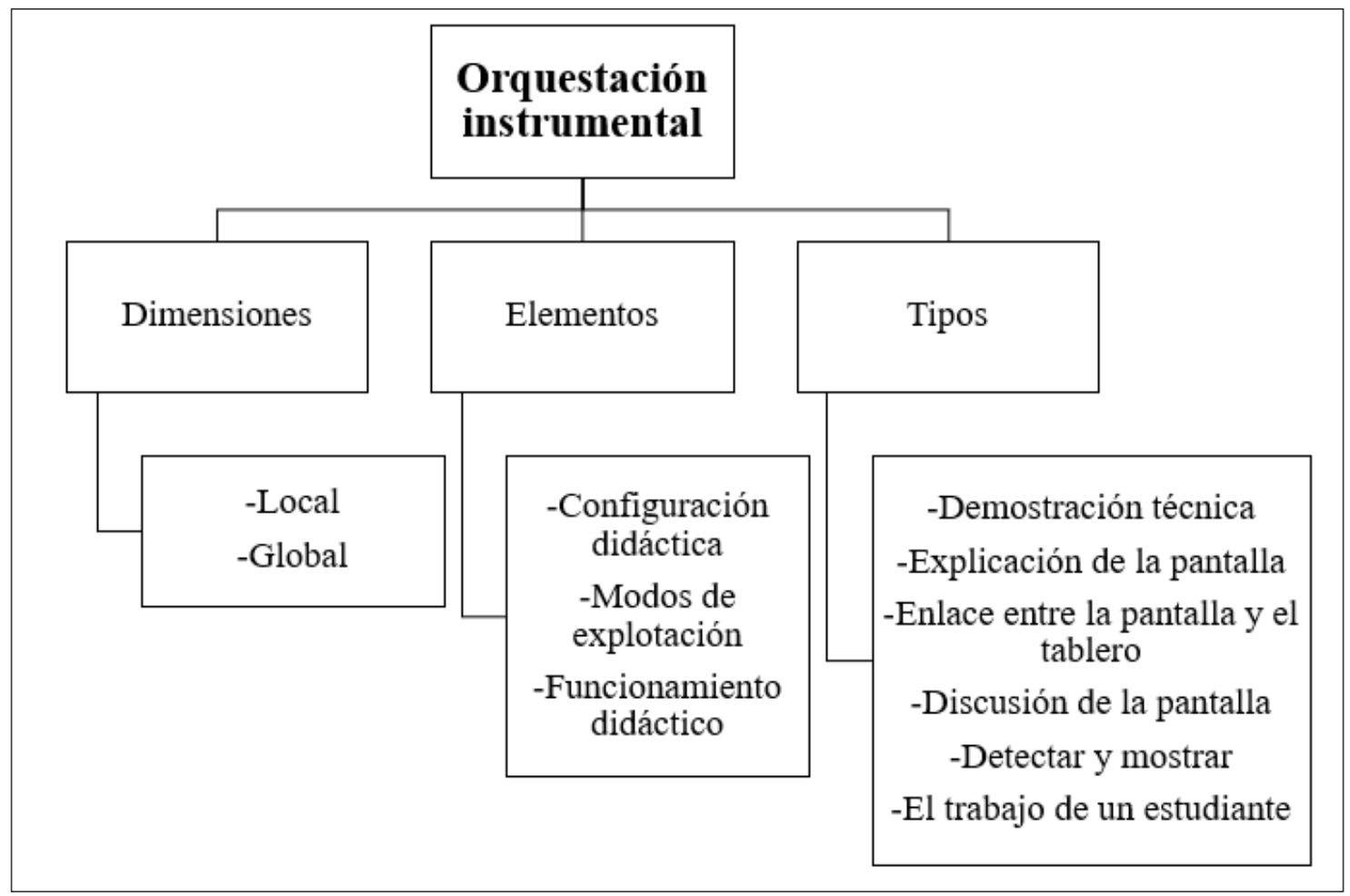

Fuente: Elaboración propia teniendo en cuenta Drijvers et al. (2010)

Como se muestra en la Figura 1 y de acuerdo con Drijvers et al. (2010), la dimensión global está referida a las técnicas de enseñanza enmarcadas en el repertorio del profesor mientras que la dimensión local está referida al contexto didáctico adaptado al grupo de estudiantes con objetivos e intenciones didácticas específicas, y es esta última la que se tendrá en cuenta en la presente investigación, ya que se aborda un concepto matemático específico impartido a un grupo de estudiantes en particular. 
En cuanto a los elementos, la configuración didáctica se refiere a la configuración de la enseñanza y los objetos o artefactos involucrados en ella; los modos de explotación, a la forma en que el profesor explota la configuración didáctica con el fin de que se cumplan las intenciones didácticas previamente establecidas y el funcionamiento didáctico se refiere a las decisiones que toma el profesor en el transcurso de la clase.

En relación con los tipos de orquestación se distinguen seis, los cuales se pueden dar por separado o por combinaciones entre algunos. Para cada tipo de orquestación existe una configuración didáctica y ciertos modos de explotación como se muestra a continuación en la Cuadro 1.

Cuadro 1: Características de los tipos de orquestación instrumental

\begin{tabular}{|c|c|c|c|}
\hline $\begin{array}{c}\text { Tipo de } \\
\text { Orquestación }\end{array}$ & Definición & $\begin{array}{l}\text { Configuración } \\
\text { Didáctica }\end{array}$ & Modos de Explotación \\
\hline $\begin{array}{l}\text { Demostración } \\
\text { técnica }\end{array}$ & $\begin{array}{l}\text { En el tipo de orquestación en } \\
\text { el que el profesor demuestra } \\
\text { las técnicas de la herramienta } \\
\text { por parte del profesor a fin de } \\
\text { evitar el surgimiento de } \\
\text { obstáculos por la falta de } \\
\text { experiencia de los estudiantes. } \\
\text { El trabajo del estudiante es } \\
\text { generalmente individual. }\end{array}$ & $\begin{array}{l}\text { El aula se debe distribuir } \\
\text { de tal forma que permita } \\
\text { a los estudiantes seguir } \\
\text { las instrucciones del } \\
\text { profesor. } \\
\text { Los estudiantes deben } \\
\text { tener acceso al applet y al } \\
\text { Entorno Digital de } \\
\text { Matemáticas (EDM) }\end{array}$ & $\begin{array}{l}\text { Los profesores pueden } \\
\text { demostrar una técnica en la } \\
\text { nueva situación o utilizar el } \\
\text { trabajo del estudiante para } \\
\text { mostrar las nuevas técnicas. }\end{array}$ \\
\hline $\begin{array}{l}\text { Explicación de la } \\
\text { pantalla }\end{array}$ & $\begin{array}{l}\text { Es el tipo de orquestación en el } \\
\text { que hay una explicación a toda } \\
\text { la clase por parte del profesor, } \\
\text { guiada por lo que sucede en la } \\
\text { pantalla del ordenador. }\end{array}$ & Similar al anterior. & $\begin{array}{l}\text { Los profesores pueden } \\
\text { tenerles trabajo a los } \\
\text { estudiantes como punto de } \\
\text { partida o empezar explicando } \\
\text { una situación con su solución. }\end{array}$ \\
\hline $\begin{array}{lcc}\text { Enlace entre la } \\
\text { pantalla } y \quad \text { el } \\
\text { tablero } & & \end{array}$ & $\begin{array}{l}\text { Es el tipo de orquestación } \\
\text { donde el profesor hace } \\
\text { hincapié en la relación entre lo } \\
\text { que ocurre en el entorno } \\
\text { tecnológico y cómo esto se } \\
\text { representa en las matemáticas } \\
\text { convencionales (lápiz y } \\
\text { papel). }\end{array}$ & $\begin{array}{l}\text { Similar al anterior. } \\
\text { Se hace uso del tablero, } \\
\text { cuaderno y un } \\
\text { computador. }\end{array}$ & $\begin{array}{l}\text { Similar al anterior. } \\
\text { Los profesores deben de } \\
\text { contribuir en la conexión de } \\
\text { las representaciones, técnicas } \\
\text { encontradas en el EDM y sus } \\
\text { contrapartes del papel y lápiz. }\end{array}$ \\
\hline $\begin{array}{l}\text { Discusión de la } \\
\text { pantalla }\end{array}$ & $\begin{array}{l}\text { Es el tipo de orquestación en el } \\
\text { que se promueve una } \\
\text { discusión con toda la clase de } \\
\text { lo que sucede en la pantalla del } \\
\text { ordenador. El objetivo es } \\
\text { mejorar la génesis } \\
\text { instrumental individual y }\end{array}$ & $\begin{array}{l}\text { Se proyecta el trabajo de } \\
\text { algún estudiante. }\end{array}$ & $\begin{array}{l}\text { Una tarea o un trabajo de los } \\
\text { estudiantes son recogidos por } \\
\text { el profesor y se discuten los } \\
\text { fenómenos más importantes } \\
\text { que ocurren en la pantalla o } \\
\text { por alguna pregunta } \\
\text { elaborada por el profesor. }\end{array}$ \\
\hline
\end{tabular}




\begin{tabular}{|c|c|c|c|}
\hline & $\begin{array}{l}\text { colectiva. Se explota el trabajo } \\
\text { previo e individual. }\end{array}$ & & \\
\hline $\begin{array}{l}\text { Detectar } \quad y \\
\text { mostrar }\end{array}$ & $\begin{array}{l}\text { Es el tipo de orquestación en el } \\
\text { que el razonamiento del } \\
\text { estudiante se pone en primer } \\
\text { plano a través de la } \\
\text { identificación del trabajo en el } \\
\text { EDM durante la preparación } \\
\text { de la lección y su uso en el } \\
\text { aula. }\end{array}$ & $\begin{array}{l}\text { Acceso al trabajo de los } \\
\text { estudiantes en el EDM } \\
\text { durante la clase. }\end{array}$ & $\begin{array}{l}\text { El profesor puede pedirles a } \\
\text { algunos estudiantes que } \\
\text { expongan sus razonamientos } \\
\text { y los demás debaten respecto } \\
\text { a esto. }\end{array}$ \\
\hline $\begin{array}{l}\text { El trabajo de un } \\
\text { estudiante }\end{array}$ & $\begin{array}{l}\text { Es el tipo de orquestación } \\
\text { donde un determinado } \\
\text { estudiante utiliza la tecnología } \\
\text { para presentar el trabajo } \\
\text { realizado o para llevar a cabo } \\
\text { las peticiones del profesor. }\end{array}$ & $\begin{array}{l}\text { El estudiante puede estar } \\
\text { al control del uso de la } \\
\text { tecnología. Los demás } \\
\text { estudiantes deben ser } \\
\text { capaces de seguir las } \\
\text { acciones del estudiante y } \\
\text { del profesor. }\end{array}$ & $\begin{array}{l}\text { El profesor puede tener un } \\
\text { trabajo presentado o se } \\
\text { explica por el sherpa- } \\
\text { estudiante, o puede plantear } \\
\text { preguntas al estudiante y } \\
\text { pedirle que lleve a cabo } \\
\text { acciones específicas en el } \\
\text { entorno tecnológico. }\end{array}$ \\
\hline
\end{tabular}

Fuente: Adaptado de Drijvers et al. (2010)

\section{Método}

El método de esta investigación asume una aproximación cualitativa, particularmente es un estudio de casos, que de acuerdo con Kothari (2004), es una manera de realizar una investigación a profundidad de una unidad social (ya sea una persona, una familia, una institución, un grupo cultural, etc.) y cuyo objetivo está centrado en localizar los factores que explican los patrones de comportamiento de la unidad dada como una totalidad integrada.

El caso es la práctica de una profesora de matemáticas cuando se implementa un diseño de tareas sobre las funciones exponencial y logarítmica que involucra GeoGebra.

En esta investigación seguimos la siguiente ruta metodológica: diseño de las tareas (basado en la teoría, en los planes de estudio y en la opinión de la profesora), colecta de datos y análisis de los datos de acuerdo con la orquestación instrumental.

Para la colecta de datos empleamos la técnica de observación no-participante ya que permitió grabar la clase (grabaciones de la clase en general y de los momentos particulares donde la profesora realizaba intervenciones) y tomar apuntes de lo que hacía la profesora y de las interacciones entre ella y los estudiantes. 


\section{Contexto de investigación y sujetos participantes}

En esta investigación, el estudio de casos fue la práctica de una profesora de matemáticas, quien es máster en educación matemática y tiene conocimiento sobre el uso de tecnología en el aula. La participación de la profesora fue voluntaria y su elección estuvo en que contaba con cocimientos sobre tecnología, lo cual era necesario para aplicar el diseño de tareas.

Para analizar la práctica de la profesora, se seleccionó uno de los grupos de estudiantes a los que ella les impartía clase de matemáticas y que se encontraran en noveno grado (año escolar en el que, de acuerdo al plan de estudios colombiano, se enseña la función exponencial). Así la implementación del diseño por parte de la profesora, se hizo en la institución donde laboraba y en el horario establecido para el área de matemáticas.

\section{Instrumento}

El instrumento fue diseñado teniendo en cuenta la teoría empleada, lo contemplado en el plan de estudio colombiano respecto a la enseñanza de la función exponencial y la opinión de la profesora. Específicamente se realizó el siguiente proceso: primero se revisaron los planes de estudio colombiano y la teoría, lo que permitió diseñar las tareas, después se le proporcionó este diseño a la profesora, quien sugirió modificaciones y finalmente se rediseñó tomando en cuenta la opinión de la profesora.

En este sentido, el diseño obtenido quedó con cuatro tareas (Hoja de tareas y applet) y tuvo por título explorando en mí entorno. La tarea 1 consta de una situación que muestra el crecimiento de una población de peces y de 9 preguntas en las que resaltó el trabajo en los registros tabular y lenguaje natural (al describir el comportamiento). La tarea 2 consta de dos preguntas relacionadas con la ubicación de los puntos hallados en la tarea 1, la graficación y validación. En esta se privilegia el trabajo en el registro algebraico y gráfico, contribuyendo así a ir ampliando el concepto de función exponencial. Estas dos tareas se encuentran en un mismo applet y su realización privilegió el uso de la herramienta de entrada que posee GeoGebra para que los estudiantes lograran dibujar los puntos y escribir la expresión matemática y así visualizaran cómo se comportaba la gráfica. Además, se da lugar a la comunicación entre pares y a la verificación de respuestas con el software. 
La tarea 3, que junto con la tarea 4 se encuentra en el segundo applet. Particularmente la tarea 3 consta de cuatro preguntas, con las que se pretendió que el estudiante pudiera emplear lo aprendido en las tareas 1 y 2 para cambiar de base (de la 2 a la 3 ) y observar el comportamiento de los dos casos gráficamente. Y la tarea 4 consta de cinco preguntas que tuvieron por objetivo que los estudiantes generalizaran lo encontrado en las tareas anteriores y llegaran así a la expresión $f(x)=a^{x}$, la introdujeran en la barra de entrada de GeoGebra, le asignaran un deslizador a $a$, lo movieran y observaran lo que sucede a fin de que caracterizaran la función exponencial.

\section{Análisis de datos}

Para el análisis de los datos colectados se elaboró una rejilla de análisis que permite caracterizar la orquestación que se lleva a cabo en cada tarea del diseño de tareas (esta rejilla es parte de una más amplia usada en una investigación que abarcaba más que la caracterización de la práctica de la profesora). La rejilla (ver Cuadro 2) ha sido conformada por unas unidades de análisis como se muestra a continuación.

Cuadro 2: Rejilla de análisis 1

\begin{tabular}{|c|c|c|}
\hline \multicolumn{3}{|c|}{ Unidades de análisis } \\
\hline \multicolumn{3}{|l|}{ Situación } \\
\hline \multicolumn{3}{|l|}{ Objetivo de la situación } \\
\hline \multicolumn{3}{|c|}{ Tarea 1} \\
\hline \multicolumn{3}{|l|}{ Tipo de tarea } \\
\hline \multicolumn{3}{|l|}{ Objetivo de la tarea } \\
\hline \multicolumn{3}{|l|}{ Tipo de orquestación } \\
\hline Configuración didáctica & Modo de explotación & Funcionamiento didáctico \\
\hline $\begin{array}{l}\text { 1. ¿Qué tipo de artefactos se usan } \\
\text { para llevar a cabo la tarea? } \\
2 . \text { ¿Cómo se distribuyen los } \\
\text { artefactos y el tiempo en el } \\
\text { desarrollo de la tarea? } \\
\text { 3. ¿Quién está al control de uso de } \\
\text { la tecnología? } \\
4 \text { ¿Qué decisiones toma la } \\
\text { profesora en relación con el diseño } \\
\text { antes de la respectiva } \\
\text { implementación? }\end{array}$ & $\begin{array}{l}\text { 1. ¿Qué elige la profesora como } \\
\text { punto de partida para el } \\
\text { desarrollo de las tareas } \\
\text { diseñadas? } \\
\text { 2. ¿De qué forma la profesora } \\
\text { muestra las estrategias de } \\
\text { solución más representativas } \\
\text { empleadas por los estudiantes? } \\
\text { 3. ¿La profesora escoge a un } \\
\text { estudiante para que exponga su } \\
\text { estrategia de solución? } \\
\text { 4. ¿Se generan discusiones } \\
\text { frente a la estrategia expuesta } \\
\text { por el estudiante? } \\
\text { 5. ¿Cómo la profesora usa el } \\
\text { software para la explicación de } \\
\text { las tareas? }\end{array}$ & $\begin{array}{l}\text { 1. ¿La profesora indica alguna forma } \\
\text { de dar respuesta a una tarea? } \\
\text { 2. ¿Qué dificultades se dan en el } \\
\text { desarrollo de las tareas? } \\
\text { 3. ¿Cómo interviene la profesora } \\
\text { cuando evidencia que el estudiante } \\
\text { resuelve inadecuadamente las tareas o } \\
\text { presenta dificultades? } \\
\text { 4. ¿Qué preguntas le plantean los } \\
\text { estudiantes a la profesora? Y ¿Cuáles } \\
\text { preguntas se socializan o debaten? } \\
\text { 5. ¿Cómo se lleva a cabo los } \\
\text { momentos de socialización o debate } \\
\text { de algunos fenómenos relevantes? }\end{array}$ \\
\hline
\end{tabular}




\begin{tabular}{|l|l|l|}
\hline & $\begin{array}{l}\text { 6. ¿La profesora incluye algún } \\
\text { artefacto diferente a los } \\
\text { propuestos? }\end{array}$ & \\
\hline
\end{tabular}

Fuente: Elaboración propia (2020).

Para el desarrollo de la investigación se tomó la investigación cualitativa como enfoque metodológico, en donde se adopta una perspectiva descriptiva-interpretativa para el análisis de los datos obtenidos de la rejilla, el cual se enmarca bajo las dimensiones establecidas por Osses, Sánchez y Ibáñez (2006). La rejilla de análisis (Cuadro 2) permitió analizar la práctica de la profesora respecto a cada elemento de la orquestación instrumental, que posteriormente dio lugar a la identificación del tipo de orquestación realizado por la profesora, tomando en consideración la caracterización de cada tipo de orquestación expuesto en el marco teórico.

Cabe señalar, que en esta investigación la configuración didáctica se empleó en la fase de diseño de las tareas, mientras que los modos de explotación y el funcionamiento didáctico se emplearon en la implementación de las tareas.

\section{Resultados}

Los resultados se presentan teniendo en cuenta los elementos y tipos de orquestación instrumental. Inicialmente se describen los elementos de la orquestación instrumental, lo cual permite hacer una comparación en lo que se esperaba que ocurriera (configuración didáctica) en el aula y lo que realmente ocurrió (modos de explotación y funcionamiento didáctico). Posteriormente, de acuerdo a estos elementos, se identifican los tipos de orquestación que la profesora realiza, lo que permite caracterizar la práctica de la profesora.

\section{Elementos de la orquestación instrumental}

\section{Configuración didáctica}

En el diseño se había establecido que las tareas se entregaran, a cada estudiante y a la profesora, en el applet y en una hoja en físico indicándole qué debía realizar. Para llevar a cabo las tareas se estipuló el uso del software GeoGebra y como hardware el computador y la hoja del estudiante. 
Cada estudiante tenía un computador y una hoja de tareas. Para el desarrollo de la tarea 1 se consideró designar un tiempo de 30 minutos, porque era la tarea en la que el estudiante se enfrentaría por primera vez al software y a la situación y además era la tarea con mayor número de preguntas; para resolver la tarea 2 se designaron 10 minutos, dado que el estudiante solo debía introducir datos en GeoGebra y verificar la expresión matemática; para la tarea 3, 10 minutos, ya que el estudiante realiza acciones similares en las tareas 1 y 2, y para la tarea 4, 30 minutos puesto que es la tarea en la que se pretende que el estudiante consolide el concepto.

Ahora bien, en las tareas 1 y 2 se pretendía que el estudiante estuviese al control de la tecnología y en las tareas 3 y 4 el control de la tecnología lo tendría la profesora, ya que era quien debía realizar la socialización y darle nombre matemático a lo encontrado, no obstante, debía brindar espacio al estudiante para explorar y resolver las preguntas.

En cuanto al uso de GeoGebra, en la tarea 1 y 4 se pretendía potencializar la herramienta del deslizador, pues mediante su uso, el estudiante podía observar la variación y cambio de la situación. Así también el uso de este software debía permitir validar las respuestas en la tarea 1 mediante el manejo de las casillas de entrada y botones, ya que al introducir el estudiante los datos, este le mostraba si el dato era correcto y en las tareas 2 y 3 mediante la introducción de los puntos y la expresión matemática, pues la gráfica correspondiente a la expresión debía pasar por los puntos.

Así, se esperaba reflejar la combinación de los tipos de orquestación Explicación de la pantalla, Detectar y mostrar y Discusión de la pantalla y que la formulación del problema se mantendría constante, pero en cada punto se aumentaba la complejidad de la tarea.

\section{Modos de explotación}

Para la implementación del diseño, la profesora lee las preguntas de la tarea 1 y explicar de manera general lo que debe hacer cada estudiante, haciendo énfasis en el uso del deslizador a fin de llevar a los estudiantes a observar lo que ocurre en el lago diariamente para que puedan responder las preguntas. Esto permitió que los estudiantes no se desviaran del propósito de la tarea.

En el diseño se había determinado un tiempo para la exploración individual por parte de los estudiantes mientras la profesora supervisaba, esto permitió que cada estudiante se enfrentara 
por sí solo a la situación, elaborando estrategias de solución con los conocimientos previos y las verificaciones del software.

La profesora realiza las siguientes preguntas a un estudiante respecto a la tarea 2 (estaban estipuladas en la hoja del profesor): ¿La gráfica coincide con los datos? y ¿Ubicaste los puntos teniendo en cuenta las coordenadas $(x, y)$ ? Estas preguntas propiciaron la reflexión por parte del estudiante en relación con el proceso que estaba llevando a cabo. Se resalta, además, que en esta tarea GeoGebra es usado por la profesora para mostrar cómo se deben de ubicar los puntos de la tabla a diferencia de las otras tareas.

La profesora tomó la decisión de propiciar un espacio para la socialización de la primera tarea, lo cual no estaba propuesto en la hoja del profesor. Dicha socialización comienza cuando la profesora considera que la mayoría de los estudiantes han realizado en su totalidad la tarea 1 , e inicia mostrando las estrategias más representativas de los estudiantes y pidiendo explicación específicamente a algunos de ellos. Las explicaciones de las estrategias se realizan en voz alta y en algunos casos los estudiantes salen al tablero.

En los momentos en que se exponen las estrategias, algunos estudiantes las refutan y en otros casos las apoyan cuando consideran que cumplen con las condiciones del problema, sin embargo, estos momentos no generan discusión alguna entre pares. Cabe señalar que, aunque se había propuesto, el estudiante no tiene la posibilidad de proyectar su trabajo, sino que solo expone verbalmente su estrategia, lo cual impide que pueda darse cuenta de cómo empleó GeoGebra para desarrollar la tarea.

En todo este proceso, se observó que la profesora empleó otros artefactos además de los propuestos, como lo es el tablero para escribir las diferentes expresiones matemáticas a las que llegaron los estudiantes y en ocasiones propuso a los estudiantes el uso de la calculadora para realizar algunos cálculos numéricos.

De manera general, se consideró que los objetivos planteados para la tarea 1 y 2 se cumplieron en su totalidad, mientras que en las tareas 3 y 4, el objetivo propuesto no se cumplió en su totalidad, debido al tiempo. 


\section{$\underline{\text { Funcionamiento didáctico }}$}

Inicialmente la profesora explicó a los estudiantes lo que debían realizar, haciendo hincapié tanto en lo que aparece en el material físico como en lo que hay en GeoGebra (ver extracto de P), esto permitió que los estudiantes pudiesen, de manera ordenada, ir dando solución a cada una de las tareas teniendo en cuenta los artefactos que se le proporcionan.

P: $\quad$ Realicen la tarea 1 bajo las respectivas premisas y paralelo a ello deben ir relacionando lo del material físico y lo que hay en el applet de GeoGebra.

Ahora bien, en el momento de la implementación surgen dificultades relacionadas con la comprensión de algunas preguntas; sin embargo, estas dificultades son minimizadas por la profesora. Por ejemplo, en la tarea 1 algunos estudiantes piden explicación de la pregunta 4, pues no sabían cómo hacer la justificación y para ello la profesora interviene como se evidencia en el siguiente extracto.

E2: ¿Cómo hago la seis?

P: $\quad$ (Lee la pregunta 6) si tengo la cantidad de peces del día 5 y en el applet no me aparece los del día 6, ¿puedo determinar la cantidad de peces del día 6 ?

E2: $\quad$ Sí.

P: ¿Cómo puedo terminar esa cantidad? ¿Por qué crees tú que puedes?

E2: Porque tenemos la información del día anterior, lo podemos duplicar o seguir la secuencia.

P: ¡Exacto! Puedo ir mirando cual es la secuencia y puedo decir que se duplica, se triplica, se multiplica por tal valor y la voy obteniendo. Eso es lo que te están preguntando, ¿cómo se obtiene eso? entonces es muy clave lo que has dicho: la secuencia. Porque no siempre es multiplicando, puede ser dividiendo, sumando o restando para obtener las cantidades, así que vas a escribir aquí tu idea sobre eso.

La profesora atendió esta dificultad mediante la explicación y la formulación de preguntas que conducen al estudiante a reflexionar en lo que está haciendo y así consolidar la respuesta. La pregunta 7 también generó dificultad por parte de los estudiantes en su comprensión, sin embargo, la profesora no explica la pregunta en relación con las intencionalidades descritas en la hoja del profesor, sino que dicha explicación la enfoca en hacerle entender al estudiante que no es posible encontrar la cantidad de peces con los datos que se le proporcionan (ver extracto de E6 y P), mostrando estar de acuerdo con los estudiantes.

E6: ¿Cómo puedo llegar al día seis a partir del día cero?

P: $\quad$ Si tengo la cantidad de peces del día cero y no conozco las demás, ¿puedo terminar cuántos peces habrá en el día seis?

E6: $\quad$ No 
P: ¿Por qué?

E6: $\quad$ No conozco la secuencia

P: La palabra clave es no conozco la secuencia...

Esta intervención y explicación de la profesora condujo a que los estudiantes se dieran cuenta que era necesario conocer la secuencia para poder responder la tarea. En este momento de socialización, los estudiantes preguntaron a la profesora acerca del porqué al elevar un número a la cero da 1 (ver extracto de E1 y P), para lo cual la profesora responde asumiendo la propiedad (pregunta del estudiante) como una definición en el campo de la matemática.

E1: ¿Por qué todo número elevado a la cero da uno?

P: $\quad$ Pues hay unas condiciones matemáticas que se tienen, eso ya es de índole estructural de los conjuntos numéricos, ¿listo?, a nosotros nos enseñan eso en la universidad para que sepamos la descomposición, pero eso hace parte del conjunto de los números, o sea hay unos teoremas, unas demostraciones que nosotros aprendemos, a ustedes no se les enseña sino la parte práctica, por eso cuando ustedes vayan en un futuro a decidir qué van a estudiar, es muy diferente ser profesor de matemáticas que ser matemático...

Así pues, de manera general la profesora atiende las dudas y dificultades de los estudiantes en los momentos de socialización lo cual permitió que se continuara con el desarrollo de las tareas. Además de esto, la profesora intervino en algunos casos en los que hubo debates entre pares conduciendo a los estudiantes a responder adecuadamente. Un ejemplo de esto es cuando una estudiante (E14) le dice a su compañera (E15) que está equivocada, pues no puede colocar y como expresión matemática, afirmando que la expresión que ella encontró es $y=2 x$. Con relación a ello la profesora les dice que no puede ser y sino que una expresión y igual a algo. La respuesta de la profesora permite, de alguna manera, aclarar dudas en relación con la sintaxis de la expresión matemática, conocimiento que deberían tener los estudiantes.

Se evidencia además que surgen diversas decisiones tomadas por la profesora, una de ellas es socializar la tarea 1 tomado las estrategias empleadas por algunos estudiantes en cada pregunta. Esta decisión fue tomada porque la profesora consideró importante escuchar las estrategias empleadas por los estudiantes, con el fin de rescatar elementos claves, llevar a los estudiantes a que compartan sus razonamientos, permitir la reflexión entre los estudiantes en relación con los resultados obtenidos y consolidar entre todos la expresión matemática que modela la situación propuesta. Aunque este espacio no estaba propuesto al finalizar la tarea 1, se considera que fue pertinente para que los estudiantes consolidaran las ideas y continuaran con las otras tareas. 
La profesora usa el trabajo de un estudiante (extracto de E2 y P) para concluir el debate sobre la expresión matemática correcta (pregunta 8), ya que con la participación de varios se logró determinar que dicha respuesta era la adecuada

P: $\quad$ E2, ¿cómo hacemos para comprobarle a estos niños que esto si cumple con esto?

E2: Como es $2^{x}$ entonces $\mathrm{x}$ representa el número de días, en cero días hay 1 pez porque cualquier número elevado a la cero da uno.

P: $\quad$ Escribámoslo allá.

E2: Cojo el dos elevado a la uno y da cero, cojo el dos elevado a la dos y da cuatro y cojo el dos elevado a la tres y da ocho y así sucesivamente.

Para mostrar mejor la estrategia mencionada en el extracto, la estudiante sale al tablero y escribe $2^{0}=1,2^{1}=2,2^{2}=4,2^{3}=8$ y paralelo realiza una tabla como se muestra en la Figura 2.

Figura 2: Respuesta en la socialización- Pregunta 8- Tarea 1

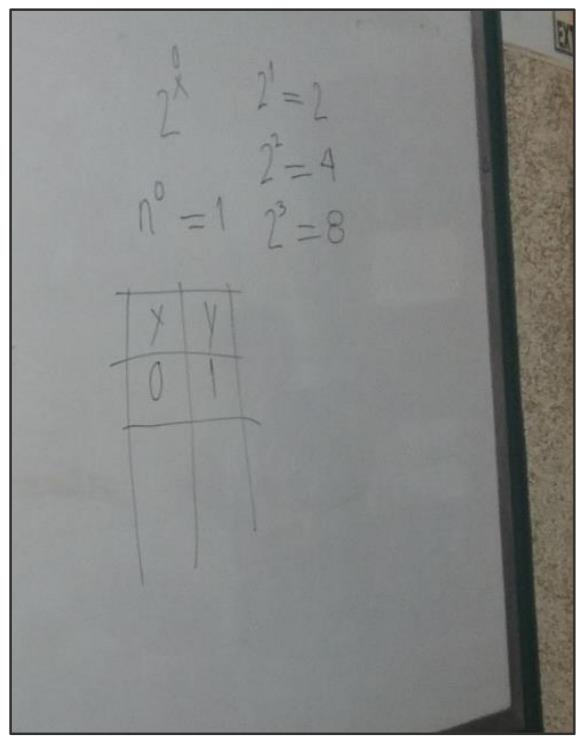

Fuente: Evidencia de la investigación

Esta socialización permitió que todos los estudiantes reconocieran la expresión matemática que modelaba los datos de la situación.

Respecto a la segunda tarea, la profesora tomó dos decisiones: una cuando dio explicaciones sobre lo que sucede en la pantalla relacionado con la tabla de la hoja de tarea, esto con el fin de que los estudiantes compararan los datos que habían completado en la tabla con los puntos que estaban obteniendo en la pantalla. La segunda decisión estuvo enfocada en la explicación dada para introducir en la barra de GeoGebra las parejas ordenadas. 
Así también, la profesora realiza a algunos estudiantes preguntas como: ¿Qué hiciste? ¿Qué te pedían? ¿Cuál fue la expresión? ¿Esos puntos de dónde los sacaste? ¿Era el $(1,2)$ el que tenías que digitar? Y ¿cuál sería tu primer punto? Las cuales les permite a los estudiantes llegar a realizar lo que se les pide en la hoja del estudiante como se observa en la Figura 3.

Figura 3: Respuesta-Pregunta 1 y 2- Tarea 2

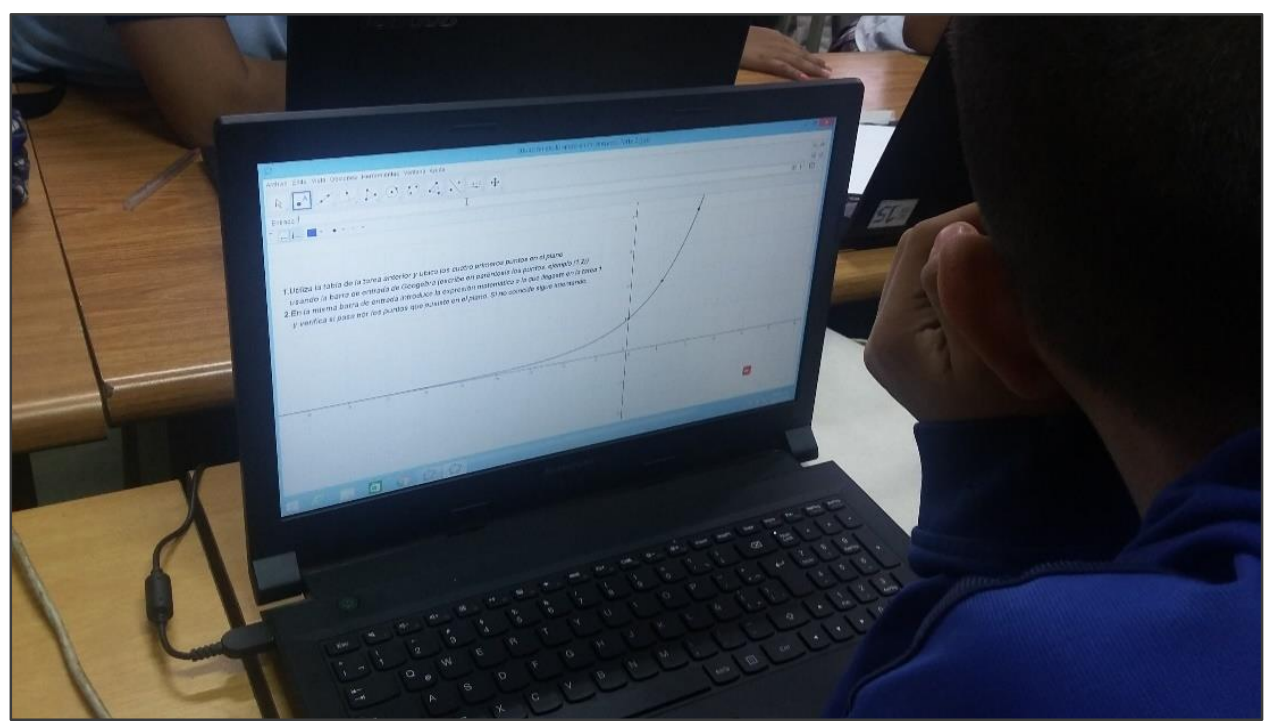

Fuente: Evidencia de la investigación

Cabe resaltar, que la profesora proporciona espacios para que haya discusión entre los mismos estudiantes, asumiendo el papel de observadora, de tal forma que esto les permita a los estudiantes corregir errores o comparar sus respuestas. Por ejemplo, en este espacio hubo dos estudiantes que se preguntan por qué la gráfica les da una recta, la cual no pasa por los puntos (ver Figura 4). Concluyendo así, que la variable x no está como exponente sino multiplicando el 2, lo que hace que corrijan y luego se den cuenta de la nueva gráfica pasa por los puntos que han introducido.

Figura 4. Respuesta 2-Pregunta 1 y 2- Tarea 2

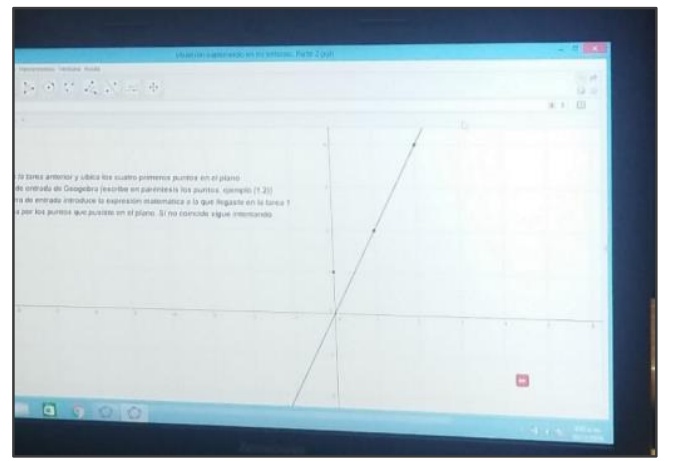

Fuente: Evidencia de la investigación 
De manera general, en esa tarea la profesora no usa las mismas estrategias que en la tarea 1, pues en esta se focaliza en intervenir de manera personalizada y así indagar a los estudiantes sobre el desarrollo de las preguntas. Respecto a las tareas 3 y 4 no se puede decir nada del funcionamiento didáctico, ya que no fueron abordadas por la profesora, debido a que el tiempo destinado fue corto.

\section{Tipos de orquestación instrumental}

La descripción realizada en los tres elementos de la orquestación instrumental, permite identificar qué tipos de orquestaciones se presentaron en la práctica de la profesora. En ese sentido, se observa que de manera general la profesora, como orquestadora, genera espacios para el trabajo individual (el estudiante trabaja con la herramienta), para la discusión entre pares, interviene en lo que hacen algunos estudiantes y de manera general en el grupo (socializando algunas tareas) realizando preguntas que contribuyeron a la reflexión de ciertos cuestionamientos.

De manera particular, se identificó en la práctica de la profesora los siguientes tipos de orquestación instrumental:

1. Enlace entre la pantalla y el tablero: se identificó cuando la profesora, en las tareas 1 y 2 , hace hincapié en la relación de lo que sucede en GeoGebra y lo que tienen los estudiantes en el material físico. En este tipo de orquestación la profesora emplea el computador, la hoja del estudiante y el tablero, estableciendo relaciones entre ellos en el momento en que se socializa la tarea 1.

2. Explicación de la pantalla: se identificó cuando la profesora tomó como punto de partida, la exploración individual de los estudiantes y posteriormente dio indicaciones de lo que los estudiantes debían realizar, haciendo uso de GeoGebra.

3. Detectar y mostrar: se da cuando la profesora gestiona un espacio para que algunos estudiantes expongan sus razonamientos y se discute alrededor de los fenómenos más importantes que se hayan encontrado en la exploración de la tarea 1.

De acuerdo a esto, se observa que la profesora no realiza un solo tipo de orquestación, sino la combinación de tres tipos, y esto es lo que caracteriza su práctica (la forma en que se llevó a cabo cada uno de los elementos y tipos de la orquestación instrumental). 


\section{Conclusiones}

De acuerdo a lo realizado en esta investigación, se puede concluir que la configuración didáctica le permitió a los investigadores y a la profesora, establecer previamente los artefactos a integrar, las intencionalidades, qué tipo de interacciones se esperaba que ocurrieran, qué conocimiento matemático se quería promover, entre otras cosas, lo cual contribuyó a que la profesora pudiese guiar de manera oportuna a los estudiantes respecto al desarrollo de cada tarea. Los modos de explotación y el funcionamiento didáctico, dieron luz de lo que realmente ocurrió en la implementación, lo cual permitió caracterizar cómo la profesora llevó a cabo cada momento en la clase mediante la integración de artefactos y cómo estos, en manos del estudiante, se convirtieron en instrumentos a través de la gestión de la profesora. Así estos elementos permitieron la identificación de los tipos de orquestación instrumental que emergieron.

La práctica de la profesora estuvo caracterizada en la combinación de tres tipos de orquestación instrumental: Enlace entre la pantalla y el tablero, Detectar y mostrar y Explicación de la pantalla. Este último tipo de orquestación, no se había contemplado en la configuración didáctica, sin embargo, fue llevado a cabo por la profesora. Por el contrario, se había contemplado el tipo Discusión de la pantalla, el cual no se identificó porque, aunque la profesora propició espacios para la socialización y el debate, no se proyectó el trabajo en GeoGebra de algún estudiante para discutir sobre este. Esto muestra, que cuando un profesor integra tecnología en el aula no emerge un solo tipo de orquestación, sino que puede darse la combinación de varios tipos.

En este sentido, se considera que emplear como categorías de análisis los elementos y tipos de orquestación instrumental permiten caracterizar la práctica del profesor cuando se integra tecnología en el aula, ya que el análisis que proporciona el uso de los elementos de la orquestación contribuye a la identificación de los tipos de orquestación instrumental que el profesor realice.

\section{Referencias}

Artigue M. (2019) Reflecting on a Theoretical Approach from a Networking Perspective: The Case of the Documentational Approach to Didactics. En: Trouche L., Gueudet G. \& Pepin B. (Eds) The 'Resource' Approach to Mathematics Education. Advances in 
Caracterización de la práctica de una profesora al implementar un diseño sobre la función exponencial...

Mathematics Education. Springer, Cham.

Castillo, L., Gutiérrez, R., \& Prieto, J. (2013). Una perspectiva de análisis de las transformaciones geométricas en curvas de la función $f(x)=e^{a x}$ utilizando GeoGebra. Revista do Instituto GeoGebra Internacional de São Paulo, 2(2), 81-92.

Castro, M., González, M., Flores, S., Ramírez, O., Cruz, M. \& Fuentes, M. (2017). Registros de representación semiótica del concepto de función exponencial. Parte I. Entreciencias: diálogos en la Sociedad del Conocimiento, 5(13), 1-12. doi: 10.21933/J.EDSC.2017.13.218.

Chevallard, Y. (1999). L'analyse des pratiques enseignantes en théorie anthropologique du didactique. Recherches en Didactique des Mathématiques, 19(2), 221-266.

Clivaz, S. \& Shuilleabhain, A. (2017). Analysing mathematics teacher learning in Lesson Study - a proposed theoretical framework. In T. Dooley y G. Gueudet (Eds.), Proceedings of the Tenth Congress of the European Society for Research in Mathematics Education (pp. 2820-2827). Dublin, Ireland: Institute of Education, Dublin City University, Ireland, and ERME.

Drijvers, P., Doorman, M., Boon, P., Reed, H., \& Gravemeijer, K. (2010). The teacher and the tool: instrumental orchestrations in the technology-rich mathematics classroom. Educational Studies in mathematics, 213-234. doi:10.1007/s10649-010-9254-5.

Ellis, A., Ozgur, Z., Kulow, T., Dogan, M. \& Amidon, J. (2016). An exponential growth learning trajectory: Students' emerging understanding of exponential growth through covariation. Mathematical Thinking and Learning, 18(3), 151-181. http://dx.doi.org/10.1080/10986065.2016.1183090.

Olsson, J. (2019). Relations Between Task Design and Students' Utilization of GeoGebra. Digital Experiences in Mathematics Education, 5(3), 223-251.

Osses, S., Sanchez, I., \& Ibañez, F. (2006). Investigación cualitativa en educación: Hacia la generación de teorías a través del proceso analítico. Estudios Pedagógicos, XXXII (1), 119-133.

Pepin, B., Choppin, J., Ruthven, K., \& Sinclair, N. (2017). Digital curriculum resources in mathematics education: foundations for change. ZDM, 49(5), 645-661. https://doi.org/10.1007/s11858-017-0879-Z.

Santos-Trigo, M. (2019). Mathematical problem solving and the use of digital technologies. In Mathematical problem solving (pp. 63-89). Springer, Cham.

Sastre, P., Cañibano, A. \& D`Andrea, R. (2017). Aplicación de la función exponencial sobre el cambio aritmético en la variable independiente. UNIÓN, (48), 84-96.

Sureda, P. \& Otero, M. (2013). Estudio sobre el proceso de conceptualización de la función exponencial. Educación Matemática, 25(2), 89-118.

Trejo, M. \& Ferrari, M. (2018). Desarrollo del Razonamiento Covariacional en Estudiantes de Nivel Medio Superior. El caso de la función exponencial. Innovación e Investigación en Matemática Educativa, 3(1), 35-58.

Trouche., L. (2002). Une approche instrumentale de l'apprentissage des mathématiques dans des environnements de calculatrice symbolique. En: Guin, D. y Trouche, D. (Eds) 
Calculatrices symboliques. Transformer un outil en un instrument du travail informatique: un problème didactique. Grenoble : La Pensée Sauvage Editions.

Trouche, L., \& Drijvers, P. (2014). Webbing and orchestration two interrelated views on digital tools in mathematics education. Teaching Mathematics and its Applications, 33(3).

Vérillon, P., \& Rabardel 1995. Cognition and artefacts: A contribution to the study of thought in relation to instrument activity. European Journal of Psychology in Education, 9(33), $77-101$.

\section{Autores}

Karen Gisel Campo-Meneses

Magíster en Docencia de la Matemática y Estudiante del Doctorado en Ciencias con Especialidad en Matemática Educativa, de la Universidad Autónoma de Guerrero. Actualmente se encuentra investigando en dos líneas: Conexiones Matemáticas y las TICs en el aula de matemáticas. Email: karencampo@uagro.mx

Gilbert Andrés Cruz Rojas

Magíster en Educación y profesor auxiliar del Área de Educación Matemática (AEM) de la Universidad del Valle y profesional del Centro de Innovación Educativa Regional Sur. Actualmente pertenece al Grupo de Educación Matemática (GEM) de la Universidad del Valle y realiza trabajos desde la línea de investigación Prácticas y Tecnologías de la Información y Comunicación en la enseñanza de las matemáticas a partir del estudio de la formación de profesores de matemáticas. Email: gilbert.a.cruz.r@correounivalle.edu.co 